\title{
GIH-17 Soluble E-cadherin is an independent pre-therapeutic factor for long term survival in gastric cancer
}

AOO Chan, KM Chu, SK Lam, BCY Wong, KF Kwok, S Law, WM Hui, YH Yueng, J Wong. Departments of Medicine and Surgery, University of Hong Kong, Queen Mary Hospital, Hong Kong.

Introduction: Gastric cancer remains the second leading cause of cancer-related deaths in the world, but a satisfactory tumor marker is currently unavailable for gastric cancer. Soluble E-cadherin has recently been found to have prognostic value in gastric cancer. We aim at evaluating whether pre-therapeutic serum soluble E-cadherin is an independent factor predicting long-term survival in gastric cancer.

Patients and Methods: 116 patients with histology proven gastric adenocarcinoma were included. Pre-therapeutic serum was collected and soluble E-cadherin was assayed using a commercially available ELISA kit. The patients were followed up prospectively at the outpatient clinic.

Results: There were 75 men and 41 women with a mean age of $66 \pm 14$ years. Forty eight percent of tumours were located in the gastric antrum. The median survival was 11 months. The mean pre-therapeutic value of soluble E-cadherin was $9159 \mathrm{ng} / \mathrm{ml}$ (range, 6002 to $10025 \mathrm{ng} / \mathrm{ml}$ ), and that of CEA was $11 \mathrm{ng} / \mathrm{ml}$ (range, 0.3 to $4895 \mathrm{ng} / \mathrm{ml}$ ). On multivariate analysis, soluble E-cadherin is an independent factor predicting long-term survival. Ninety percent of patients with serum level of E-cadherin greater than $10000 \mathrm{ng} / \mathrm{ml}$ had survival less than 3 years $(\mathrm{P}=0.009)$.

Conclusions: Soluble E-cadherin is a potentially valuable pre-therapeutic prognostic factor in patients with gastric cancer.

\section{GIH-18 PIN1 is over-expressed in hepatocellular carcinoma (HCC) and correlates with an increased beta-catenin and cyclin D1 protein levels}

\author{
Roberta Pang, John Yuen*, M.F. Yuen*, C.L. Lai*, Y.L. Kwong and E. Tse \\ Divisions of Haematology/Oncolgy, and Hepatology*, Department of Medicine, University of Hong Kong
}

Introduction: In hepatocellular carcinoma, the expression of beta-catenin and cyclin D1 is increased, which may be of pathogenetic significance. As mutations of the beta-catenin gene are only found in around $20 \%$ of cases, other factors are involved in the accumulation of beta-catenin and cyclin D1. PIN1, a peptidyl-proplyl-isomerase, has been shown to stabilize both beta-catenin and cyclin D1, and to up-regulate cyclin D1 gene expression. We hypothesize that the beta-catenin and cyclin D1 accumulation in some of the HCC is contributed by PIN over-expression.

Methods: The expression of PIN1 in 23 paired-samples of neoplastic and non-neoplastic liver tissues was examined by semi-quantitative reverse transcription polymerase chain reaction (RT-PCR), immunohistochemistry and Western blot analysis. Immunohistochemistry was also performed on another 28 paired archival samples of HCC to detect PIN1, beta-catenin and cyclin D1 expression.

Results: Compared with paired non-neoplastic liver tissues, 12 of 23 (52\%) HCC samples showed an increase in PIN1 expression by semi-quantitative RT-PCR. These cases also showed beta-catenin accumulation, and sequencing of exon 3 of the beta-catenin gene did not show any mutation. Together with the archival materials, PIN1 was found to be over-expressed by immunohistochemistry and Western blot analysis in 26 of 45 tumors (58\%), all of which had concomitant accumulation of beta-catenin. Another 5 cases had beta-catenin accumulation without PIN1 over-expression, so that the overall frequency of beta-catenin over-expression was $68 \%$ (31/45). In 3 cases with beta-catenin accumulation but no PIN1 over-expression, 2 cases showed mutation in the exon 3 of the beta-catenin gene. Finally, 19 of 26 cases with PIN1 over-expression also had increase in cyclin D1 expression.

Conclusion: PIN1 expression is increased in a significant proportion of HCC. There is a positive correlation between PIN1, and beta -catenin and cyclin D1 expression, which suggests that PIN1 may be critically involved in hepatocarcinogenesis. 